\title{
Light-coupling masks: An alternative, lensless approach to high-resolution optical contact lithography
}

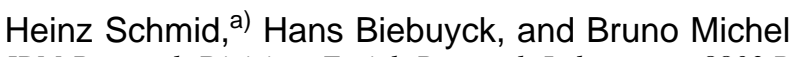 \\ IBM Research Division, Zurich Research Laboratory, 8803 Rüschlikon, Switzerland \\ Olivier J. F. Martin and Nicolas B. Piller \\ Institute for Field Theory and Microwave Electronics, Swiss Federal Institute of Technology (ETH), \\ 8092 Zurich, Switzerland
}

(Received 29 May 1998; accepted 16 September 1998)

\begin{abstract}
We describe an approach to optical lithography using light-scattering contact masks with protruding elements that couple light into a photoresist. This method differs from conventional contact lithography in two important ways. First, because portions of the light-coupling mask (LCM) are made from a polymer, intimate contact with the resist occurs over large areas without additional load. This contact is readily reversible, and causes no observable damage or contamination of the LCM or substrate. Second, the structure formed by the protruding parts of the LCM in contact with the resist can define local optical modes that impart directionality to the light propagating through the LCM and amplify its intensity. We provide an experimental realization and theoretical description of the method, demonstrating its use for the formation of $100 \mathrm{~nm}$ features with light having a wavelength of $256 \mathrm{~nm}$. (C) 1998 American Vacuum Society. [S0734-211X(98)09806-0]
\end{abstract}

\section{INTRODUCTION}

Contact lithography with metal on glass masks ${ }^{1,2}$ has been replaced by projection lithography for high-resolution patterning, despite its simplicity and economy. Contact lithography uses comparably simpler illumination sources without sophisticated imaging optics while maintaining large fields of exposure. Two problems limit its application however: One is the difficulty of placing two hard and somewhat fragile objects, the chrome-patterned quartz mask and the resistcoated wafer, in intimate contact over large areas. Damage and contamination are typical when high contact forces must be applied to eliminate air gaps originating from even minute deviations from substrate flatness. Second, as the feature size on the mask shrinks below the exposed wavelength, the amount of transmitted light is small and diffracted into high angles, reducing the achievable depth of exposure. The lightcoupling-mask (LCM) approach ${ }^{3}$ alleviates these problems by using a structured polymer to contact the resist (Fig. 1). The polymer is able to make a conformal contact with the substrate because it can accommodate small height variations on the substrate without additional applied load. The contact is reversible without damage to the substrate or polymer so that the LCM can be used many times without suffering mechanical wear. The side of the LCM that is in contact with the resist is structured in height according to the desired pattern. Structure sizes equal to or less than the exposing wavelength act like waveguides that can couple light into the resist with high directionality and intensity, thereby allowing the exposure of small structures with high contrast.

a) Author to whom correspondence should be sent; electronic mail: sih@zurich.ibm.com

\section{PRINCIPLE OF LIGHT-COUPLING MASKS}

The intensity distribution of light leaving a structured mask in contact with a substrate varies with the size of its structures and the details of the implementation. In what follows we provide a qualitative, idealized illustration of some of the phenomena relevant to the determination of how light exits a LCM. We use a schematic representation of its lightcoupling structures. Theoretical models using self-consistent analyses of the behavior of electric fields in a continuum dielectric provide additional and quantitative support of this technique, and will be described below.

Several conditions are defined in our description. First, we assume that light enters the LCM from its backside as an isointensity, monochromatic plane wave with circular polarization perpendicular to its axis of propagation. The ratio of structure width to height is set to unity, reflecting a plausible case for mechanical stability ${ }^{4}$ of features in a polymeric mask. Both the refractive index of the LCM and that of the photoresist are set to be the same (1.6). When light enters a LCM (Fig. 1) the matrix causes a blueshift that is propor-

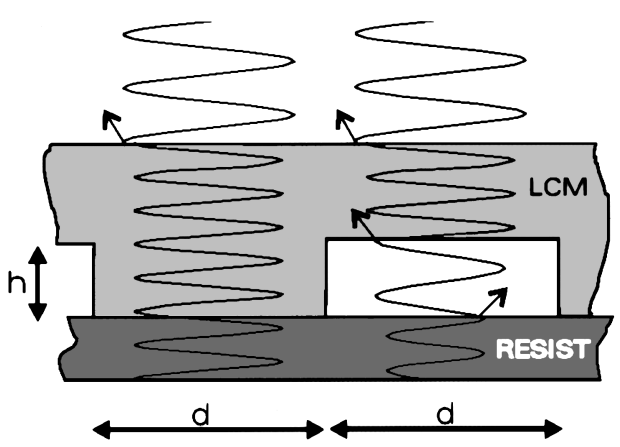

FIG. 1. Schematic of light passing through a structured polymer in contact with a resist. 
tional to the refractive index of the polymer in the effective wavelength of the traveling wave. The path of light is noticeably altered as the wave front encounters structured boundaries in the mask. The degree of change and the principal cause of contrast in the intensity of light as it exits the LCM relate to the boundary and its scale relative to the wavelength $\lambda$ in the polymer. Three different regimes illustrate the origin of the contrast in LCMs, in which the width of the structure is $\lambda \ll d, \lambda \lesssim d$, and $\lambda>d$.

In the first case, $\lambda \ll d$, incoming light splits into two paths at the LCM structure. Along the path at the left-hand side (Fig. 1), light travels within the polymeric structure and is transmitted into the resist without significant reflection losses. Light traveling the path to the right-hand side must, on the other hand, cross an air gap and two boundaries with substantial index mismatch. Two different types of contrast result. First, a strong phase contrast occurs below the edges of structures in the mask by destructive interference in the resist where light from the two paths begins to overlap. Maximal contrast of this type results if the phase delay between the two beams is $\pi$, as demonstrated by Rogers et al. ${ }^{5}$ forming a derivative image of the mask structures in the resist (i.e., edges of the mask structures are not exposed). Second, contrast occurs by reflection losses experienced by light on the different paths, as shown in Fig. 1. LCMs operate in this regime. The change in amplitude can be used to form a 1:1 image of the mask structure in the top layer or in a thin resist layer. Figure 2(a) shows an attempt to expose a thick, positive resist where the reflection contrast was insufficient for an accurate exposure: light leaked through the air gap and exposed the underlying resist. In addition, phase contrast to the light intensity distorted the desired amplitudederived image. This situation is clearly unfavorable and demonstrates the need to suppress the phase-shifted path. One way to improve contrast in LCMs with features where $\lambda$ $\ll d$ is to place an absorbing or reflecting layer or structure in the air gaps. We used the selective placement of a 10-nmthick gold film, as illustrated in Fig. 2(b). ${ }^{3}$ LCMs of this type allowed an accurate 1:1 reproduction of large mask features in thick resist at the price of a more difficult realization of the LCM.

Results of computer simulations shown in Fig. 3(a) ${ }^{6}$ explain the origin of the contrast in a LCM for a case where $\lambda>d$. Light passing the center structure follows the path of higher polarizability, effectively focused there by the overall structure, which leads to a strong forward directionality in the light propagation. The figure shows that standing waves exist above the air gaps of the LCM, and that some of the energy associated with this field is redirected into the lightcoupling structure and the resist. Thus these smaller mask features in the LCM act more like short waveguides with zero-order modes, confining light and causing local amplification of its intensity so that the contrast is enhanced compared to the reflection case. Such small structures in a LCM avoid phase contributions to the exposure of resist because the intensity difference of light traveling along the two paths (as in Fig. 1) is large. The phase difference of the two waves

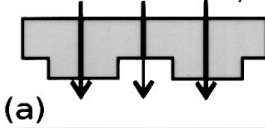

(a)

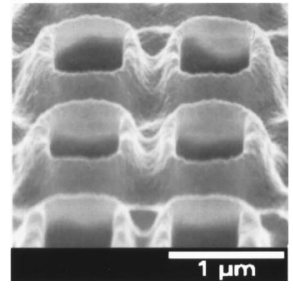

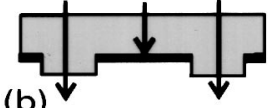

(b)

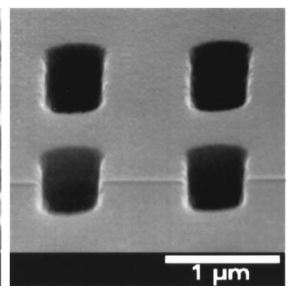

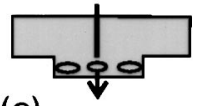

(c)

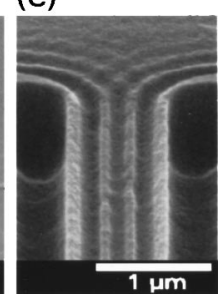

FIG. 2. Schematic of the light path through various LCMs having structures with $\lambda \ll d$ (top), and examples of the resulting exposures in resist (bottom). (a) Light insufficiently blocked by the air gap contributes an undesirable contrast to the image in the positive resist. (b) Differential placement of a 10 nm gold layer on this LCM allows accurate exposure of its features. (c) Near-field image of the consequences of diffraction modes excited within an LCM having $\lambda \leqslant d$, visualized by exposure of a negative resist. The wavelength of the source for all exposures was $365 \mathrm{~nm}$. The height of the structure in the LCM was $270 \mathrm{~nm}$. The positive resist was AZ6612 (Hoechst), and the negative resist na410 (Micro Resist Technology).

is, in addition, small because the air gap is now only a fraction of the wavelength. The confinement and penetration depth of the field created by the converging-lens effect of the air gap is improved compared to the case where only a metallic absorber is used to define an aperture, as illustrated in Fig. 3(b). The metallic aperture significantly attenuates the light that reaches the resist and acts more like a divergentlens element, leading to stronger diffraction and spreading of the light field, as would be the case for an idealized, conventional contact mask. The improved contrast and forward di-
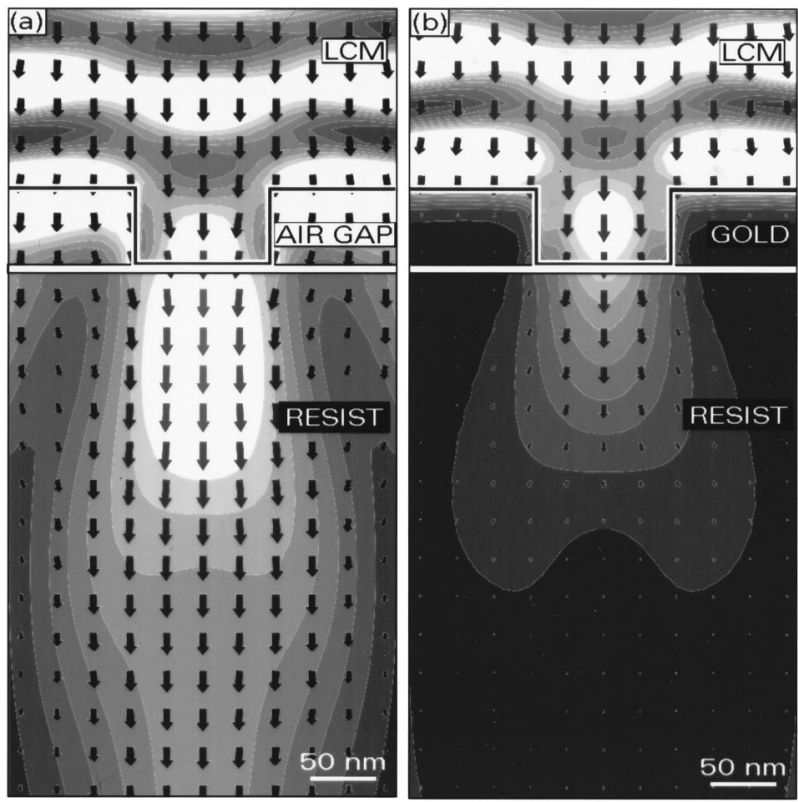

FIG. 3. Simulation of the propagation of $248 \mathrm{~nm}$ light through (a) a $100 \mathrm{~nm}$ wide LCM with $60 \mathrm{~nm}$ high air gaps or (b) an aperture defined by $60 \mathrm{~nm}$ thick metal layers as in an idealized, classical contact mask (Ref. 6). Contours are isointensity lines from a three-dimensional simulation scaled in increments of $10 \%$, where white represents the highest intensity. Arrows show the time-average Poynting vector that gives the energy flow in the structure. Input intensity is the same in each case. 
rectionality of light leaving a LCM compared to that of a chrome mask makes high-resolution exposure of a resist possible. Small mismatches in the refraction indices between the LCM and the resist cause additional reflections from the contacting area but did not, in our experiments, affect the outcome significantly. Simulations, as well as experiments (Fig. 4) also show that the resolution of LCMs is wavelength limited. Structures smaller than about half the wavelength in the polymer do not accurately confine light as the field leaks out into the surrounding air, reducing spatial resolution.

In the intermediate region with mask features where $\lambda$ $\varsigma d$, all phase-, amplitude-, and mode-contrast mechanisms contribute to the image formation. In the latter mechanism, light confined within structures larger than the wavelength begins to interfere with itself, exciting higher-order modes with intensity minima that manifest themselves in the resist, [Fig. 2(c)]. If these minima prove too disturbing, interferences within the LCM structure and the resulting near-fielddiffraction patterns in the resist ${ }^{7}$ can be blurred using multiple wavelengths or broadband exposures where the smallest wavelength sets the resolution. Given these considerations, Fig. 5 demonstrates that sufficient contrast is nonetheless available to expose through a 480-nm-thick resist with features equal to or larger than the exposing wavelength. The exposure depth available for an accurate transfer was $\approx 100$ $\mathrm{nm}$ in this case, as judged from the sidewall profile, favoring top surface imaging or a bilayer scheme ${ }^{8}$ for pattern transfer.

Simulations also show that polarization-related phenomena can be substantial for some structure geometries at small scales, although this case has not yet been explored experimentally.

\section{MATERIAL REQUIREMENTS AND IMPLEMENTATION}

Conformity and transparency of LCMs are the principal constraints of their design. Conformal contact between two substrates without the addition of an applied load requires some degree of flexibility or adaptiveness at their interface. Elastomeric polymers adhering to a variety of hard substrates demonstrate this condition. Besides adaptiveness, the material of a LCM must also be capable of useful structural delineation so that features defined on its surface remain viable and robust to contact, remaining intact after release from a photoresist. Such mechanical strength and hardness are clearly at odds with the formerly stated requirements of conformality of the material, and restricts the range of useful systems for implementation. The range of useful systems is further restricted by dimensional accuracy, which requires low-temperature cure and low thermal expansivity. The final requirement on LCMs is about their transparency. LCMs must guide light into the resist, ideally by the processes of elastic light scattering in the matrix as discussed above. In the case of LCMs, this requirement is fortunately somewhat less stringent than for classical optical elements because the polymer layer can be made relatively thin while maintaining the requisite of other attributes of LCMs. Furthermore, random-light-scattering effects inside the LCM, associated

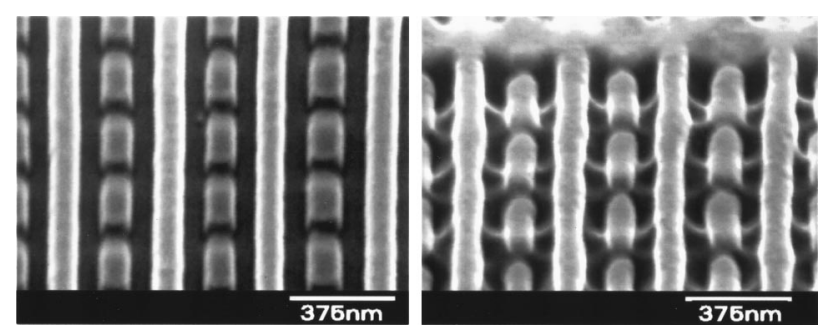

FIG. 4. Exposure at the resolution limit of an LCM using light centered at $256 \mathrm{~nm}$ with a $40 \mathrm{~nm}$ bandpass. The LCM was replicated from a silicon master with $120 \mathrm{~nm}$ lines separated by $70 \mathrm{~nm}$ and a depth of $80 \mathrm{~nm}$ (lefthand side) and used to expose a positive resist (UV5, Shipley) (right-hand side), proving the success of the overall replication strategy at this scale. No additional absorbers were used to enhance the contrast of the LCM in this case.

with heterogeneities or other types of imperfections in the material, are to some extent tolerable as light tends to be strongly guided within the structures. We find that filled materials comprising a polymer and inorganic particles that scatter light such as silica (used for strength) are thus also capable of forming useful LCMs.

In the work reported here, the exposed light had wavelengths well into the ultraviolet (UV) range. The general absorbance of organics in this part of the spectrum rules out many typical, all-carbon elastomers such as poly(butadiene). We focused on the formulation of siloxane-based materials ${ }^{9}$ that have sufficient transmission at $256 \mathrm{~nm}$ and yield relatively strong materials (Young's modulus up to $15 \mathrm{MPa}$ ). The refractive index of the polymer used was $\sim 1.45$. Stable structures down to $100 \mathrm{~nm}$ form in this material and withstand the rigors of their formation and their use as LCMs.

More macroscopic considerations about the fabrication of LCMs are important too. Practically, the avoidance of air trapped between the LCM and the substrate is one key to facilitating their contact. The use of a vacuum between the two is one obvious solution. More simply, a slight bow ${ }^{10}$ added to the mask neatly allows propagation of the contact front. Separation of the LCM and substrate can also be non-

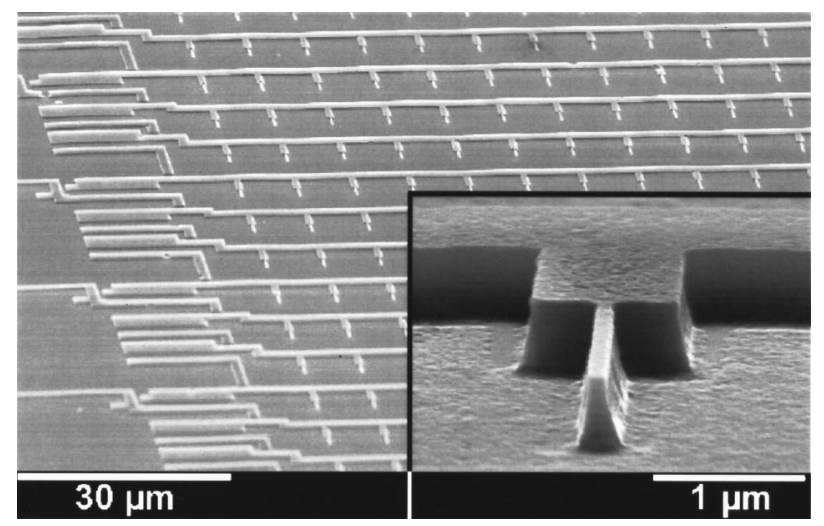

FIG. 5. Positive resist (UV5, Shipley) exposed with an LCM having a mixture of small $(120 \mathrm{~nm})$ and large $(>1 \mu \mathrm{m})$ features and using light centered at $256 \mathrm{~nm}$ with a $40 \mathrm{~nm}$ bandpass. The inset demonstrates the achievable contrast and resist profile. Here, the structure height of the LCM was 100 $\mathrm{nm}$, and no additional absorbers were used to enhance its contrast. 

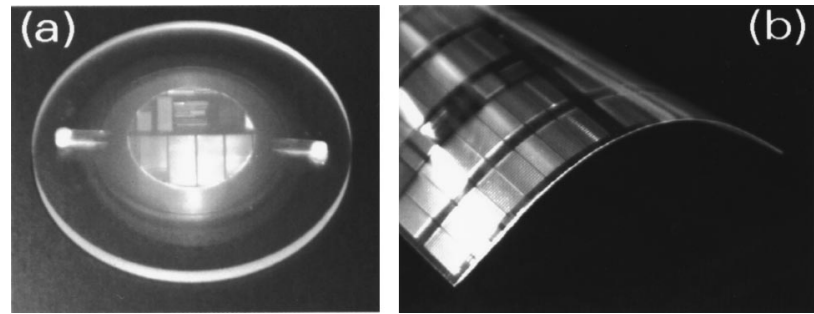

FIG. 6. Two possible implementations of LCMs. (a) Multilayer LCM on a rigid, 30-mm-diam quartz backplane with an intermediate, elastomeric layer $(\approx 1 \mathrm{~mm}$ thick, having a Young's modulus of $\approx 3 \mathrm{MPa})$ and structured siloxane layer on top ( $20 \mu \mathrm{m}$ thick, having a Young's modulus of $\approx 30$ MPa). (b) LCM made with a structured polymer layer directly supported on a polymer foil.

trivial. The relatively large work of adhesion between them, a quality of the conformality of the LCM and substrate, prevents simple, simultaneous release. Better is the nucleation of release from one edge, effectively focusing the shear stress in a peeling-like action. Such release favors some capacity for bending of the LCM or substrate and the use of thin elastomeric layers, particularly when the LCM must cover very large areas $\left(>10 \mathrm{~cm}^{2}\right)$.

We investigated several approaches to the fabrication of LCMs. The simplest LCMs were made by casting the prepolymer over a silicon master with a 5-nm-thick, plasmadeposited fluorocarbon layer using thermo- or UV-cured siloxanes to form a 1-2-mm-thick layer that was subsequently pealed away from the master. ${ }^{11}$ LCMs of this type can be used to test the mechanical and optical properties of the polymer material. We are currently also evaluating more sophisticated, multilayer approaches for LCMs to be used for lithographic application, where dimensional stability over large areas and alignability are essential, as illustrated in Fig. 6(a). These LCMs consist of three layers: a mechanically and thermally stable quartz backplane, an elastomeric intermediate layer $(0.1-1 \mathrm{~mm}$ thick) which allows compensation of imperfect wafer flatness, and on top a structured polymer layer (1-50 $\mu \mathrm{m}$ thick). The structured layer can also be supported directly on a glass or polymer foil, allowing some degree of flexibility. ${ }^{2}$ LCM based on thin glass backplanes shows the smallest pattern distortion ${ }^{9}$ because the polymer layer can be made very thin [Fig. 6(b)]. The overall transparency of LCMs allows an assessment of their relative position either in proximity to or in contact with the substrate, facilitating alignment. These hybrid approaches look promising and practical in our initial surveys and will certainly be worth further refinement.

\section{SUMMARY}

This work focused on the demonstration and simulation of the LCM principle by means of filtered coupled-dipole approximation and a self-consistent solution to the light- scattering problem. Theory predicts that LCMs can replicate patterns in thin resists with a resolution limited to about half of the smallest wavelength in the polymer $\left(\lambda_{\mathrm{vac}} / 2 n\right)$. The image is generated by a structured conformal contact mask that guides and couples light directly into the resist. For this new type of conformal contact we make use of the work of adhesion of the polymer to the substrate so that the material "grabs" the surface and does not have to be "forced" onto the surface by vacuum as in the case of conventional contact lithography. The second main difference is the light guiding effect of the polymer that allows favorable exposure of the resist. We demonstrated the strength of this new soft contact lithography approach by replication of $100 \mathrm{~nm}$ patterns over large areas using a $256 \mathrm{~nm}$ light. Exposed patterns in the resist match with the simulated intensities and demonstrate that the method works close to the theoretically predicted limit.

The ease, simplicity, and reliability of soft contact lithography will allow low-cost patterning below the micron level. It is not clear, at this point, which applications will be most promising but we foresee fabrication of gratings, filters, and holograms, all requiring pattern replication close to or below light wavelengths.

\section{ACKNOWLEDGMENTS}

The authors thank our colleagues A. Beck, A. Bernard, A. Bietsch, E. Delamarche, and H. Rothuizen for their help and discussions. They are particularly indebted to H. R. for his lithography expertise and E. Kiewra for his help with the silicon masters. One of the authors (O.J.F.M.) gratefully acknowledges the funding of the Swiss National Science Foundation. They also thank P. Guéret (IBM) and R. Vahldieck (ETH Zurich) for their support of the project. Two of the authors (B.M.) and (H.B.) acknowledge partial support from the Swiss Federal Office for Education and Science within the ESPRIT basic research program NANOWIRES (23238).

${ }^{1}$ E. Cullmann, Semicond. Int. 8, 332 (1985).

${ }^{2}$ H. I. Smith, Rev. Sci. Instrum. 40, 729 (1969).

${ }^{3}$ H. Schmid, H. Biebuyck, B. Michel, and O. J. F. Martin, Appl. Phys. Lett. 72, 2379 (1998).

${ }^{4}$ E. Delamarche, H. Schmid, B. Michel, and H. Biebuyck, Adv. Mater. 9, 741 (1997).

${ }^{5}$ J. A. Rogers, K. E. Paul, R. J. Jackman, and G. M. Whitesides, Appl. Phys. Lett. 70, 2658 (1997).

${ }^{6}$ O. J. F. Martin and N. P. Piller, Phys. Rev. E 58, 3909 (1998); O. J. F. Martin, C. Girard, and A. Dereux, Phys. Rev. Lett. 74, 526 (1995); for an animation of these results, see O. J. F. Martin et al., Opt. Express 7, 280 (28 Sept. 1998) (on line).

${ }^{7}$ J. Aizenberg, J. A. Rogers, K. E. Paul, and G. M. Whitesides, Appl. Phys. Lett. 71, 3773 (1997).

${ }^{8}$ Special issue on Optical Lithography, IBM J. Res. Dev. 41, No. 1/2 (1997).

${ }^{9}$ H. Schmid and B. Michel (preprints available).

${ }^{10}$ E. Cullmann, K. Cooper, and C. Reyerse, Suss Report (Karl Suss America, Waterbury Center, VT, 1991), Vol. 5, No. 3, p. 1.

${ }^{11}$ Y. Xia and G. M. Whitesides, Angew. Chem. Int. Ed. Engl. 37, 550 (1998). 